\title{
Sinais e sintomas de violência sexual infantojuvenil: relatos de profissionais de saúde
}

\author{
Signs and symptoms of children's sexual violence: reports of health professionals \\ Señales y síntomas de la violencia sexual infantojuvenil: relatos de profesionales de la salud
}

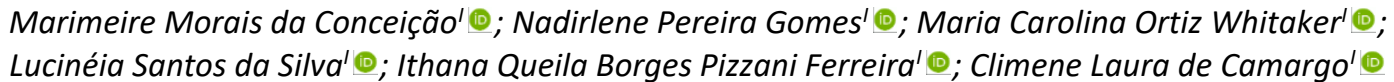

'Universidade Federal da Bahia, Salvador, BA, Brasil

\begin{abstract}
RESUMO
Objetivo: identificar sinais e sintomas apresentados por crianças e adolescentes que despertam nos profissionais de saúde a suspeita/identificação de violência sexual. Método: estudo com abordagem qualitativa realizado em 2019, com 30 profissionais de um hospital público da Bahia, mediante gravação das entrevistas com base em questionário semiestruturado e analisadas segundo Bardin. O projeto foi aprovado pelo Comitê de Ética em Pesquisa. Resultados: dor, sangramento, alteração ou laceração da genitália e/ou ânus e Infecções Sexualmente Transmissíveis constituem sinais e sintomas que despertam os profissionais para suspeita e/ou identificação da vivência de violência sexual infantojuvenil. Conclusão: os profissionais de saúde suspeitam e/ou identificavam a vivência de violência sexual quando crianças e adolescentes apresentam complicações relacionadas ao trato genital e anal, que podem resultar em óbito.
\end{abstract}

Descritores: Criança; Adolescente; Delitos Sexuais; Sinais e Sintomas.

\section{ABSTRACT}

Objective: to identify signs and symptoms presented by children and adolescents that lead health personnel to suspect or identify sexual violence. Method: this qualitative study was conducted in 2019, with 30 health personnel from a public hospital in Bahia, by recording interviews based on a semi-structured questionnaire, which were analyzed by reference to Bardin. The project was approved by the research ethics committee. Results: pain, bleeding, alteration or laceration of the genitalia and/or anus and sexually transmitted infections are signs and symptoms that lead health personnel to suspect and/or identify that children and adolescents have experienced sexual violence. Conclusion: health personnel suspected and/or identified the experience of sexual violence when children and adolescents had complications relating to the genital and anal tract, which can result in death.

Descriptors: Child; Adolescent; Sexual Offenses; Signs and Symptoms.

\section{RESUMEN}

Objetivo: identificar señales y síntomas presentados por niños y adolescentes que despiertan la sospecha / identificación de violencia sexual en los profesionales de la salud. Método: estudio con enfoque cualitativo realizado en 2019, junto a 30 profesionales de un hospital público de Bahía, mediante el registro de las entrevistas en base a un cuestionario semiestructurado y analizadas según Bardin. El proyecto fue aprobado por el Comité de Ética en Investigación. Resultados: dolor, sangrado, alteración o laceración de los genitales y / o ano e Infecciones de Transmisión Sexual son señales y síntomas que hacen sospechar y / o identificar, a los profesionales, la experiencia de violencia sexual contra niños y adolescentes. Conclusión: los profesionales de la salud sospechan y / o identifican la experiencia de violencia sexual cuando los niños y adolescentes tienen complicaciones relacionadas con el tracto genital y anal, que pueden resultar en la muerte.

Descriptores: Niño; Adolescente; Delitos Sexuales; Signos y Síntomas.

\section{INTRODUÇÃO}

A violência sexual (VS), ou abuso sexual, é um agravo experienciado por crianças e adolescentes em diversos países e culturas. No âmbito internacional, a Organização Mundial de Saúde (OMS) estima que, em todo o mundo, uma em cada cinco mulheres sofreu VS na infância ${ }^{1}$ e estudo multicêntrico realizado no Malawi, Quênia, Camboja, Haiti e Tanzânia alerta que a prevalência de VS contra adolescentes varia, nestes países, entre 82 e $93 \%{ }^{2}$. Inquérito nacional constatou que, dos 2.575.269 adolescentes escolares brasileiros entrevistados, 101.901 foram forçados a ter relações sexuais somente no ano de $2015^{3}$.

Salienta-se que esses números não expressam a real dimensão desse agravo, uma vez que os registros representam apenas uma parcela do quantitativo de crianças e adolescentes que sofrem VS. A principal causa dessa subnotificação pode estar relacionada com o silêncio da vítima infantojuvenil, em decorrência do medo ao sofrer retaliações, ameaças proferidas pelo agressor, de desestruturar as relações familiares ou que sua fala não receba credibilidade dos parentes, o que ocorre com frequência na VS incestuosa ${ }^{4-6}$. 
Outra situação que corrobora para a subnotificação da VS, é a dificuldade de confirmação do fato, considerando que nem sempre existe o contato físico entre o agressor e a vítima. Estudo demonstra que mulheres vitimizadas na infância e/ou adolescência foram frequentemente expostas a imagens e cenas de cunho sexual, ou alvos de toques e carícias feitas pelos agressores ${ }^{6}$. Este tipo de VS, apesar do poder de provocar perturbações mentais, não deixa marcas visíveis. Contudo, não significa dizer que abusos que ocorrem sem penetração genital sejam menos danosos para os indivíduos, sobretudo, por estarem relacionados a quadros depressivos, cutting e ideação suicida ${ }^{4,7}$.

A este respeito, é importante pontuar que, grande parte da produção do saber nessa temática, centra-se nas sequelas psicológicas do abuso em detrimento das consequências para o corpo físico, inclusive referente a risco iminente de morte ${ }^{8-11}$. No entanto, os sinais físicos são relevantes no processo de identificação dos abusos, ainda que não sejam os únicos aspectos que devam gerar suspeita e investigação dos fatos.

Em que pese, estudo transversal realizado com 156 crianças e adolescentes peruanos abusados sexualmente, com idade entre um e 15 anos de idade, tenha evidenciado que, menos de $40 \%$ das vítimas apresentam sinais específicos e, em apenas $3 \%$, nota-se a presença de lesões em genitália ${ }^{11}$, e investigação no Instituto Médico Legal (IML) da Bahia pontua que em quase $90 \%$ dos casos de VS os médicos não conseguem identificar indícios da violação ${ }^{12}$. Sabe-se que muitas vítimas apresentam sintomatologias causadas pelo abuso e que são passíveis de observação nas unidades de saúde.

Assim, é essencial que em todo e quaisquer serviços de saúde à crianças e adolescentes, os profissionais estejam sensíveis e preparados(as) para a identificação da VS, possibilitando oferecer-Ihes tratamento humanizado. Evita-se, assim, que retornem ao ambiente hostil em que vivem e sejam tratados livres de constrangimentos, conforme recomenda o Estatuto da Criança e do Adolescente (ECA) ${ }^{13}$

Considerando a magnitude da VS contra crianças e adolescentes e o desafio que envolve sua identificação, intervenções para o reconhecimento precoce e cuidados necessários, é necessário persistir na compreensão do fenômeno tão antigo - e ao mesmo tempo tão atual, inclusive que demandam por atendimento de maior complexidade.

Buscando responder a seguinte questão norteadora: Que sinais e sintomas, apresentados por crianças e adolescentes, despertam nos profissionais de saúde a suspeita e/ou identificação de violência sexual?, este estudo apresenta como objetivo: Identificar que sinais e sintomas apresentados por crianças e adolescentes despertaram nos profissionais de saúde a suspeita/identificação de violência sexual, tendo por finalidade promover o cuidado que abrange a proteção de crianças e adolescentes vitimizados(as).

\section{MÉTODO}

Trata-se de um estudo de abordagem qualitativa, desenvolvido em um hospital público do estado da Bahia, Brasil, que atende indivíduos de todas as faixas etárias, nas diversas especialidades, incluindo urgência/emergência. Previamente, o projeto foi apresentado para as coordenações da equipe multiprofissional do referido hospital, para fins de divulgação da execução da pesquisa.

A coleta de dados ocorreu presencialmente, desenvolvida entre os meses de junho e julho de 2019, nos períodos matutino, vespertino e noturno em unidades de Emergência Pediátrica, Centro Cirúrgico, Centro Obstétrico, Terapia Intensiva e de Internação Clínica-cirúrgica. Durante a coleta, a equipe de entrevistadores(as) abordou os(as) profissionais nas unidades assistenciais, apresentou-se e, caso o(a) profissional atendesse aos critérios de inclusão (ser profissional de saúde e estar atuando por, no mínimo, um ano na instituição), era convidado a participar da pesquisa. Não houve recusas proferidas pelos participantes.

A partir do aceite de participação, um segundo encontro era agendado para realizar a entrevista. As participantes responderam à seguinte questão aberta: Como você suspeitou/identificou a vivência de VS por crianças e adolescentes atendidos? As entrevistas foram realizadas por integrantes de um grupo de pesquisa da Escola de Enfermagem da Universidade Federal da Bahia (EEUFBA), devidamente treinados. As entrevistas, gravadas em um celular Android, com durabilidade de oito a 45 minutos, foram encerradas devido à saturação dos dados.

As falas foram transcritas e seu conteúdo analisado pelo modelo sugerido por Bardin ${ }^{14}$, cumprindo a análise em três etapas: a) pré-análise; b) exploração do material; c) tratamento dos resultados e interpretação. A técnica permitiu a avaliação e interpretação dos dados mediante leitura flutuante, seguida de leituras repetidas e detalhadas do material transcrito. Os registros de um diário de campo feitos, após cada entrevista pelos pesquisadores, também foram agregados às análises temáticas, gerando códigos fornecidos mediante uma árvore de codificação. Após a sistematização dos dados, surgiram temas agrupados por semelhança de conteúdo que originaram cinco categorias temáticas: Dor; Sangramento; Alteração na genitália e/ou ânus; Laceração na genitália e/ou ânus e Infecções Sexualmente Transmissíveis (IST). 
A organização deste estudo foi embasada na ferramenta Consolidated Criteria for Reporting Qualitative Research (COREQ). O projeto foi aprovado pelo Comitê de Ética e Pesquisa da instituição dos pesquisadores e do hospital locus do estudo, visando atender às normas éticas de pesquisa com seres humanos de acordo com as Resoluções do Conselho Nacional de Saúde. Para tanto, foi elaborado um Termo de Consentimento Livre e Esclarecido (TCLE) assinado após ser lido pelas participantes selecionadas. As entrevistas ocorreram em salas que garantiam a privacidade das informações. Para proteger a identidade das participantes, seus nomes foram substituídos pela letra "P" (profissional) e por números de 1 a 30, referente, de acordo com a ordem de realização das entrevistas.

\section{RESULTADOS}

A amostra intencional constou de 30 participantes, todas do sexo feminino, sendo 12 técnicas/auxiliares de enfermagem, dez enfermeiras, três médicas, três assistentes sociais e duas psicólogas. Todas autorreferidas de identidade de gênero mulher cis, orientação afetivo-sexual heterossexual, $60 \%$ pardas e $20 \%$ pretas. Do total, $63 \%$ afirmaram ter um ou mais filhos e $47 \%$ declaram-se solteiras.

$\mathrm{Na}$ experiência de profissionais que atuam nas unidades hospitalares, alguns sinais e sintomas os despertam a suspeita/investigação de abuso sexual em crianças e adolescentes. São elas:

\section{Dor}

As profissionais entrevistadas apontam a dor em região genital como um dos sintomas apresentados por crianças e adolescentes que remeteu à suspeita/investigação de VS, conforme as narrativas:

Era um menino de um ano de idade que estava com muita dor na região do ânus e ele chorava muito!! [...] a equipe suspeitou de VS por causa da região acometida. (P2, Auxiliar de Enfermagem)

Havia registro no prontuário dela de lesão vaginal. [...] suspeitou-se de VS porque a adolescente estava com muita dor na vagina, inclusive precisou ser sedada para o médico fazer a avaliação. (P17, Enfermeira)

O sinal de dor é uma condição que serviu de alerta para as profissionais e, ao passo que a vitima localiza esta sensação dolorosa, as profissionais da saúde foram capazes de levantar suspeitas da exposição ao abuso com intercurso sexual.

\section{Sangramento}

Conforme relatos das profissionais, o sangramento genital e/ou anal consiste em um dos sintomas da VS a crianças e adolescentes atendidos na unidade hospitalar em estudo, como ilustrado pelas falas:

[...] era uma bebê, com menos de dois anos. Identificamos como VS porque ela chegou com uma hemorragia pela vagina. (P8, Técnica de Enfermagem)

Atendi alguns casos de VS: um menino de um ano e seis meses com a fralda cheia de sangue e uma adolescente com sangramento vaginal intenso, com a hemoglobina muito baixa. (P2, Auxiliar de Enfermagem)

A partir desses relatos, pode-se inferir que a localização do sangramento serve de embasamento para o diagnóstico de VS.

\section{Alteração na genitália e/ou ânus}

As alterações presentes em genitais e/ou em região perinal de crianças e adolescentes também foram descritas pelas profissionais como indícios de VS, como identificado nos relatos:

[...] enquanto a Enfermagem preprava o corpo, observei que o ânus da criança estava muito grande, aquele não era o tamanho normal de um ânus de uma criança!! O médico falou que a criança poderia ter sido abusada constantemente e que, apesar de não haver dilaceração, perfurações, presença de sangue, poderia haver uma hemorragia interna que levou à parada cardiorespiratória irreversível e o óbito, mas isso só descobriria no IML. (P27, Psicóloga)

A mãe trouxe a criança ao hospital porque ela estava com a vagina bastante avermelhada, inchada. [...] nesse caso é uma situação de abuso sexual mesmo. (P12 Assistente social)

As profissionais utilizam de um conhecimento prévio do que consideram normal para a anatomia da genitália. A partir dessa premissa relativizam que algo de errado aconteceu ocasionando a mudança percebida.

\section{Laceração na genitália e/ou ânus}

As lacerações em região genital/ânus percebidas pelas profissionais entrevistadas também foram elencadas enquanto sinais de VS sofrida por crianças e adolescentes.

Lembro de uma criança que a genitália estava toda machucada, dilacerada. Você não conseguia identificar o que era ânus e o que era vagina. (P23, Técnica de Enfermagem) 
[...] foi constatada nessa adolescente ruptura do fundo do Saco de Douglas, ocasionado, provavelmente, pela introdução de um objeto pontudo ou pela penetração à força. Apesar disso, ela não queixava de dor [...] em outro caso, atendi um menino que a região perianal estava muito ferida, muito machucada, tinha queloides, cicatrizes anteriores e fístulas, ou seja, a VS acontecia há algum tempo. (P2, Auxiliar de Enfermagem)

As profissionais revelam que, diante da condição clínica grave inclusive com acometimento de órgãos mais internos, as vítimas deveriam sinalizar sensação dolorosa, um pressuposto que está relacionado com o aprendizado formal, porém nem sempre é condizente com a realidade.

\section{Infecções sexualmente transmissíveis (IST)}

Sintomatologias relaciondas às ISTs também foram mencionadas como sinal de vivência de VS por criancas/adolescentes que foram admitidas na unidade hospitalar:

A adolescente foi sequestrada e a encontraram com secreções vaginais, mas ela não tinha nenhuma marca no corpo, nenhum sinal de agressão. Os médicos acreditam que ela adquiriu a IST do abusador. (P19, Técnica de Enfermagem)

A mãe relatou que a adolescente foi abusada e os médicos logo suspeitaram que ela contraiu a IST do agressor. (P30, Enfermeira)

Esta realidade observada pelas profissionais em estudo revela que a IST pode ser um achado clínico relacionado à exposição à violência sexual. Também, nota-se que os relatos das profissionais apontam que, nem todas as crianças e adolescentes apresentavam marcas físicas (hematomas, arranhões e lesões superficiais) e dor no corpo. Com isso, podese inferir que, nem sempre, o agressor sexual de crianças e adolescentes provoca esses danos à vítima, motivo pelo qual o exame físico completo e a escuta sensível precisam ser usadas por profissionais da saúde para identificar casos de violência sexual nesse público.

\section{DISCUSSÃO}

A suspeita/identificação da VS contra crianças e adolescentes se dá a partir de sinais e sintomas, a exemplo de sangramento, alterações e lacerações na genitália, infecções sexualmente transmissíveis, bem como a dor.

A sensação dolorosa é um sinal fisiopatológico decorrente de estímulos que se transformam em impulsos que alcançam o cérebro, onde são interpretados como dor ${ }^{15}$. Demonstrada mediante expressões corporais, verbalizações, gemidos, contrações musculares e choro, a dor indica para cuidadores(as), que há algo errado com infante/adolescente, sendo essencial a investigação com vistas na descoberta da sua origem. Considerada sinal vital, a dor expressa por crianças e adolescentes pode ser indício de VS, a dor pode ser física e emocional, localizada ou inespecífica. Quando na região da genitália e/ou anal, a dor ocorre porque crianças e, muitas vezes, adolescentes, não têm condição biopsicoemocional desenvolvida para atos sexualizados ${ }^{16,17}$.

Estudos versam que pessoas vitimizadas na infância e adolescência apresentam sensação de dor emocional e física durante e após a exposição à VS, resultando em sofrimento que vem acompanhado de sentimento de culpa e vergonha4 ${ }^{6}$. Estes dados sugerem que o mal-estar e a dor não física também podem ser sinal de vivência de violência. Sendo assim, profissionais da saúde necessitam de preparo emocional e conhecimento dessas informações, posto que sinais inespecíficos apresentados por crianças e adolescentes precisam ser profundamente investigados, a fim de compreender se estão correlacionados com abusos sexuais.

Os agressores sexuais infantojuvenis, sobretudo os que têm relação de parentesco, iniciam suas investidas com carícias, jogos de sedução que ludibriam a vítima e a convencem de serem amadas e desejadas pelo autor, como identificam estudiosos ${ }^{6,16}$. Tais sentimentos são prazerosos às vítimas, que não se percebem em situação de abuso, e podem ser a justificativa para a supressão da sensação dolorosa em casos de VS.

Esse modus operandis do agressor sexual de crianças e adolescentes conta com um conjunto de artimanhas que evoluem brincadeiras, gestos e toques sexualizados, que podem aos poucos serem direcionadas às partes íntimas, porém sem penetração genito-genital $\left.\right|^{1,6,14,18}$.

Levando em consideração a desproporcionalidade entre os órgãos genitais de adultos e a genitália/ânus de crianças/jovens, o ato sexual com penetração nesses órgãos pode ser danoso e provocar lacerações nas vítimas, como foi observado neste estudo. Corroborando, estudos internacionais apontam a predominância de casos com lesões genitais perceptíveis, que são resultantes do contato físico entre agressor sexual e vítima ${ }^{11,19,20}$. Da mesma forma, a literatura científica internacional mostra que a laceração anal é um dos danos acarretados às vítimas, sendo uma evidência de agressão sexual contra crianças em tenra idade ${ }^{21,22}$.

Estudo com 1.500 meninas entre zero e 17 anos que foram abusadas e realizaram exame anogenital com imagens digitais, observou que, das meninas examinadas após 72 horas do episódio, 2,2\% tinham achados físicos da VS como 
trauma agudo e/ou hematomas, ou mesmo o rompimento do hímen, e, entre as examinadas antes das 72 após a VS, a prevalência dessas lesões foi de $21,4 \%$, de modo que, quanto mais rápido a vítima for avaliada, maior precisão no diagnóstico ${ }^{18}$. Assim, cabe uma observação e anamnese atenta, até porque alterações menores na genitália, como hiperemias, prurido e edemas, podem estar presentes em crianças que usam fraldas e/ou engatinham, como resultantes de pequenos traumas advindos da higiene, da interação de cremes e fluidos com a pele (processos alérgicos), ou mesmo a dermatite de contato da área das fraldas.

Outra consequência apontada pelo estudo remete a IST em crianças e adolescentes, conforme apontam estudos que versam sobre implicações da $\mathrm{VS}^{22-25}$, à exemplo de pesquisa peruana com crianças e adolescentes na qual, das 156 vítimas estudadas, $25 \%$ foram acometidas por IST ${ }^{11}$. Situações como essas podem ser associadas à VS, pois a presença de alguns microrganismos, como a tricomoníase, ocasionam agravos e são passíveis de identificação por familiares e profissionais, visto que muitas apresentam secreção genital com odor e aspecto característicos, conforme asseveram estudiosos $22,24-26$.

Outro sinal apontado pelas profissionais apresentado por crianças e adolescentes que chegam à unidade e que gera suspeita/investigação de VS consiste no sangramento. Este é o sinal mais visível que permite inferir a existência de trauma com abertura dos vasos e, consequente, extravasamento sanguíneo. Estudo realizado entre 1984 e 2014 em um serviço de atendimento a crianças e adolescentes, aponta que a presença sangramento genital intenso é causa da ida de vítimas de VS às emergências, muitos demandam uso de anestésicos para a realização do exame físico, utilizado para evitar que as vítimas revisitem as sensações e lembranças ocasionadas pelo trauma dos procedimentos ${ }^{27}$.

Conforme fora percebido pelas colaboradoras deste estudo, diferentes alterações físicas são sugestivas de vivência de VS infantojuvenil. Apesar disso, muitas vezes, pequenas lesões não chocam tanto profissionais e/ou familiares, como ocorre com as rupturas de períneo e outras lesões de gravidade considerável, que demonstram maior necessidade de recorrer aos serviços de saúde. Importante pontuar, contudo, que lesões graves, como a ruptura do fundo de Saco de Douglas ou traumas e lacerações de regiões como ânus, vagina e perianal, observadas pelas profissionais neste estudo, representam somente $1 \%$ dos 156 casos de VS contra menores de 15 anos avaliados em estudo internacional ${ }^{11}$.

As lesões decorrentes da VS podem ainda ocasionar outras consequências tais quais disfunções sexuais, genitourinárias crônicas, perda da sensibilidade táctil, perdas sanguíneas anormais ou intensas e óbito, como fora observado em estudos prévios ${ }^{4,8,27-28}$. Denota-se, portanto, que as consequências físicas da VS oriundas do trauma de órgãos externos e internos interferem negativamente nas condições físicas e comportamentais de crianças e adolescentes, podendo incidir na vida das vítimas de forma fatal, conforme também assinalado nas narrativas das profissionais entrevistadas, quando referem sobre casos de meninos e meninas cujo óbito foi associado à vivência de VS, ainda que alguns casos somente após constatação do óbito.

A morte por crime sexual contra crianças e adolescentes ainda é uma ocorrência que choca a sociedade, porém este não é um fenômeno raro de ocorrer. Estudiosos apontam que um percentual significativo de registros de infanticídio em um país africano estão relacionados com a VS, onde 52,3\% das vítimas encontram-se na faixa etária entre um e 18 anos, sendo que a agressão sexual está associada a um caso em cada 10 mortes ${ }^{8}$. No âmbito nacional, pesquisa acerca de 23.278 casos de agressão contra mulheres registrados no SINAN, entre 2011 e 2015, constatou que, crianças (entre 0 e 9 anos) vítimas de estupro apresentam risco 159 vezes maior de serem assassinadas; para adolescentes (entre 10 e 19 anos) esta taxa é de 28,8 vezes ${ }^{29}$.

No sentido de reduzir a morbimortalidade por abusos sexuais em crianças e adolescentes, o estudo alerta para a necessidade de inspecionar as alterações em genitália e/ou ânus, sangramento anormal e aspectos, como a eliminação urinária (enurese) e dejeções (encoprese), além de estar atentos(as) a ações cotidianas, como alimentar-se, marchar, sentar e sinais como a reatividade a ações normais do cuidado, como abertura das pernas para trocar fraldas.

Para acolher casos de VS infantojuvenil requer conhecimento e comunicação com rede de apoio e serviços especializados, posto que as vítimas podem necessitar de acompanhamento para além da idade adulta, bem como da execução de um plano de cuidados que atente para suas necessidades e especificidades. Essas ações denotam a importância de profissionais sensíveis e preparados para suspeita e identificação da violência contra crianças e adolescentes, bem como atuação diante desse evento, conforme alertam estudos ${ }^{17,22,30-32}$. Fica evidente a necessidade de gestores atuantes em unidades hospitalares e da rede básica de saúde o desafio de estruturar e implementar protocolos no atendimento a crianças e adolescentes que incluam a VS.

Considerando o compromisso de toda sociedade para o enfrentamento da VS infantojuvenil, cidadãos e profissionais não devem abster-se de realizar denúncias e notificações, mesmo em situações suspeitas ${ }^{1,13,31,32}$. A problemática em questão requer implementação de políticas públicas existentes ${ }^{32}$, novas e distintas investigações 
científicas, incluive focalizando familiares que cuidam destas crianças, bem como profissionais de serviços especializados para fins de comparação dos resultados.

Além disso, recomenda-se a inserção nas matrizes curriculares, em projetos extensionistas para socialização do conhecimento. Sugere-se ainda que todas as ações relacionadas à prevenção da violência sexual, cuidados e proteção às vítimas infantojuvenis sejam feitas no âmbito da saúde, educação (creches, orfanatos e escolas) e segurança, no sentido de promover a capacitação dos cidadãos na prevenção e/ou identificação da VS, ainda que em situações cotidianas.

\section{Limitações do estudo}

O estudo se deu apenas com profissionais de saúde de um único hospital público, o que pode influenciar na caracterização clínica apresentada pelas vítimas; as ocorrências identificadas são sugestivas de abuso sexual e não como fatos concretos, pois o estudo não acompanhou as decisões judiciais. Sugerimos, pois, a replicação deste estudo em hospitais da rede particular e de casos julgados como VS.

\section{CONCLUSÃO}

O estudo revela que profissionais entrevistadas que atuam em serviço hospitalar suspeitam e/ou identificam a VS quando crianças e adolescentes apresentam complicações relacionadas ao trato genital e anal, como dor, sangramento, alteração ou laceração da genitália e/ou ânus e IST, que podem resultar em óbito.

Em que pese este estudo limite-se por não ter confirmada juridicamente a ocorrência dos abusos, tampouco suas cirscuntâncias, assim o estudo promove reflexões importantes quanto à responsabilidade profissional no trato com estas crianças e adolescentes, que não deve restringir-se ao cuidado às demandas clínicas que justificam o atendimento, abrangendo a investigação de suas causas e de abusos domésticos, inclusive sexuais.

\section{REFERÊNCIAS}

1. World Health Organization (WHO). Global status report on violence prevention 2014. 2015. Available from: https://apps.who.int/iris/bitstream/handle/10665/145087/WHO_NMH_NVI_14.2_eng.pdf?sequence=3.

2. Stark L, Seff I, Hoover A, Gordon R, Ligiero D, Massetti G. Sex and age effects in past-year experiences of violence amongst adolescents in five countries. Plos One [Internet]. 2019 [cited 2018 Jul 06]; 14(7):e0219073. DOI: https://doi.org/10.1371/journal.pone.0219073.

3. Fontes LFC, Conceição OC, Machado S. Childhood and adolescent sexual abuse, victim profile and its impacts on mental health. Ciênc. saúde coletiva [Internet]. 2017 [cited 2018 Jul 06]; 22(9):2919-28. DOI: https://doi.org/10.1590/141381232017229.11042017.

4. Lira MOSC, Rodrigues VP, Rodrigues AD, Couto TM, Gomes NP, Diniz NMF. Sexual abuse in childhood and its repercussions in adult life. Texto contexto - enferm [Internet]. 2017 [cited 2018 Jul 06]; 26(3):e0080016. DOI: http://dx.doi.org/10.1590/010407072017000080016.

5. Adinew YM, Mekete BT, Adinew YM. Testimony of an Ethiopian girl sexually assaulted by her stepfather: a case report. J Med Case Reports [Internet]. 2018 [cited 2018 Jul 06]; 12:231. DOI: https://doi.org/10.1186/s13256-018-1777-x.

6. Fornari LF, Sakata-So KN, Egry EY, Fonseca RMGS. Gender and generation perspectives in the narratives of sexually abused women in childhood. Rev. latinoam. enferm. [Internet]. 2018 [cited 2018 Jul 06]; 26:e3078. DOI: http://dx.doi.org/10.1590/15188345.2771.3078.

7. Correia CM, Gomes NP, Diniz NMF, Andrade ICS, Romano CMC, Rodrigues GRS. Child and adolescent violence: oral story of women who attempted suicide. Rev. Bras. Enferm. [Internet]. 2019 [cited 2018 Jul 06]; 72(6):1450-6. DOI: https://doi.org/10.1590/00347167-2017-0814.

8. Abrahams N, Mathews S, Lombard C, Martin LJ, Jewkes R. Sexual homicides in South Africa: A national cross-sectional epidemiological study of adult women and children. Plos One [Internet]. 2017 [cited 2018 Jul 06]; 12(10):e0186432. DOI: https://doi.org/10.1371/journal.pone.0186432.

9. Cisse $C T$, Niang MM, Sy AK, Faye EH, Moreau JC. Aspects épidémiocliniques, juridiques et coût de la prise en charge des abus sexuels chez les mineurs à Dakar, Sénégal. J Gynecol Obstet Biol Reprod [Internet]. 2015 [cited 2018 Jul 06]; 44(9):825-31. DOI: DOI: https://doi.org/10.1016/j.jgyn.2014.10.010.

10. Afifi TO, Macmillan HL, Boyle M, Taillieu T, Cheung K, Sareen J. Child abuse and mental disorders in Canada. CMAJ [Internet]. 2014 [cited 2018 Jul 06]; 186(9):324-32. DOI: https://doi.org/10.1503/cmaj.131792.

11. Veirano C, Zunino C, Pandolfo S, Vomero A, Guerrero J, Gurin L et al. Hospital admission to hospital due to child abuse. Pereira Rossell Hospital Center 2010-2014. Arch. PeArch. Pediatr. [Internet]. 2017 [cited 2018 Jul 06]; 88(5):246-53. Available from: http://www.scielo.edu.uy/scielo.php?script=sci_arttext\&pid=S1688-12492017000500246. 
12. Silva WS, Ribeiro FM, Guimarães GK, Santos MS, Almeida VPS, Barroso-Junior UO. Factors associated with child sexual abuse confirmation at forensic examinations. Ciênc. saúde coletiva. [Internet]. 2018 [cited 2018 Jul 06]; 23(2):599-606. DOI: https://doi.org/10.1590/1413-81232018232.04932016.

13. Castro EG, Macedo SC. Estatuto da Criança e Adolescente e Estatuto da Juventude: interfaces, complementariedade, desafios e diferenças. Rev. Direito Práx. [Internet]. 2019 [cited 2018 Jul 06]; 10(2):1214-38. DOI: https://doi.org/10.1590/21798966/2019/40670

14. Bardin L. Análise de Conteúdo. 20th ed. São Paulo: Edições 70; 2016.

15. Ellison DL. Physiology of Pain. Crit. Care Nurs. Clin. North Am. [Internet]. 2017 [cited 2018 Jul 06]; 29(4):397-06. DOI: https://doi.org/10.1016/j.cnc.2017.08.001.

16. Antony S, Almeida EM. Vítimas de violência sexual intrafamiliar: uma abordagem gestáltica. Rev. NUFEN. [Internet]. 2018 [cited 2018 Jul 06]; 10(2):184-201. Available from: http://pepsic.bvsalud.org/scielo.php?script=sci_abstract\&pid=S2175$25912018000200012 \& \operatorname{lng}=e s \& n r m=i s o \&$ tlng=pt.

17. Mathews B, Collin-Vézina D. Child Sexual Abuse: Toward a Conceptual Model and Definition. TVA [Internet]. 2019 [cited 2018 Jul 06]; 20(2):131-48. DOI: https://doi.org/10.1177/1524838017738726.

18. Gallion HR, Milam LJ, Littrell LL. Genital Findings in Cases of Child Sexual Abuse: Genital vs Vaginal Penetration. J Pediatr Adolesc Gynecol [Internet]. 2016 [cited 2018 Jul 06]; 29(6):604-11. DOI: https://doi.org/10.1016/j.jpag.2016.05.001.

19. Varma S, Gillespie S, McCracken C, Greenbaum VJ. Characteristics of child commercial sexual exploitation and sex trafficking victims presenting for medical care in the United States. Child Abuse Negl [Internet]. 2015 [cited 2018 Jul 06]; 44:98-105. DOI: https://doi.org/10.1016/j.chiabu.2015.04.004.

20. Enyedy A, Tsikouras P, Csorba R. Medical and Legal Aspects of Child Sexual Abuse: A Population-Based Study in a Hungarian County. Int. J. Environ. Res. Public Health [Internet]. 2018 [cited 2018 Jul 06]; 15(4):701. DOI: https://doi.org/10.3390/ijerph15040701.

21. Karabağ G, Tanrıverdi Hi, Yavuz MS, Genç A, Akın U, Saraç S. The importance of clinical approach in aggravated sexual abuse: Case report. Ulus Travma Acil Cerrahi Derg [Internet]. 2018 [cited 2018 Jul 06]; 24(6):597-00. DOI: https://doi.org/10.5505/tites.2018.60063.

22. Vrolijk-Bosschaart TF, Brilleslijper-Kater SN, Benninga MA, Lindauer RJL, Teeuw AH. Clinical practice: recognizing child sexual abuse-what makes it so difficult? Eur J Pediatr [Internet]. 2018 [cited 2018 Jul 06]; 177:1343-50. DOI: https://doi.org/10.1007/s00431-018-3193-z.

23. Nunes MCA, Lima RFF, Morais, NA. Violência Sexual contra Mulheres: um Estudo Comparativo entre Vítimas Adolescentes e Adultas. Psicol. cienc. prof. [Internet]. 2017 [cited 2018 Jul 06]; 37(4):956-69. DOI: https://doi.org/10.1590/19823703003652016.

24. Crawford-Jakubiak JE, Alderman EM, Leventhal JM. Committee on child abuse and neglect; committee on adolescence. Care of the Adolescent After an Acute Sexual Assault. Pediatrics. [Internet]. 2017 [cited 2018 Jul 06]; 139(3):e20164243. Erratum in: Pediatrics [Internet]. 2017; 139(6). DOI: https://doi.org/10.1542/peds.2016-4243.

25. Adams JA, Farst KJ, Kellogg ND. Interpretation of Medical Findings in Suspected Child Sexual Abuse: An Update for 2018. J. Pediatr. Adolesc. Gynecol. [Internet]. 2018 [cited 2018 Jul 06]; 31(3):225-31. Erratum in: J. Pediatr. Adolesc. Gynecol. [Internet]. 2018;31(6):655. DOI: https://doi.org/10.1016/j.jpag.2017.12.011.

26. 26. Kotzé JM, Brits H. Child sexual abuse: The significance of the history and testifying on non-confirmatory findings. Afr J Prim Health Care Fam Med [Internet]. 2019 [cited 2018 Jul 06]; 11(1):e1-e7. DOI: https://doi.org/10.4102/phcfm.v11i1.1954

27. Abraham M, Kondis J, Merritt DF. Case Series: Vaginal Rupture Injuries after Sexual Assault in Children and Adolescents. J Pediatr Adolesc Gynecol [Internet]. 2016 [cited 2018 Jul 06]; 29(3):e49-52. DOI: https://doi.org/10.1016/j.jpag.2015.12.009.

28. Gibson CJ, Lisha NE, Walter LC, Huang AJ. Interpersonal trauma and aging-related genitourinary dysfunction in a national sample of older women. Am J Obstet Gynecol [Internet]. 2019 [cited 2018 Jul 06]; 220(1):94.e1-94.e7. DOI: https://doi.org/10.1016/j.ajog.2018.09.026.

28. Barufaldi LA, Souto RMCV, Correia RSB, Montenegro MMS, Pinto IV, Silva MMA et al. Gender violence: a comparison of mortality from aggression against women who have and have not previously reported violence. Ciênc. saúde coletiva [Internet]. 2017 [cited 2018 Jul 06]; 22(9):2929-38. DOI: https://doi.org/10.1590/1413-81232017229.12712017.

29. Conceição MM, Ferreira BO, Barreto ETP, Nery, CLPD, Santos JSNT, Camargo CL. Perceptions of a multidisciplinary team on the psychological repercussions of sexual violence against children and adolescents. Texto Contexto Enferm [Internet]. 2021 [cited 2021 nov 23]; 30:e20200500. DOI: https://doi.org/10.1590/1980-265X-TCE-2020-0500.

30. Reis, NSP et al. Scientific production in nursing on violence in adolescence. Rev. enferm. UERJ [Internet]. 2017 [cited 2018 Jul 06]; 25:e26475. DOI: https://doi.org/10.12957/reuerj.2017.26475.

31. Amarijo, Cristiane Lopes et al. Assimilating the theory and practice of domestic violence: nursing professionals providing primary care for victims. Rev. enferm. UERJ [Internet]. 2018 [cited 2018 Jul 06]; 26:e33874. DOI: https://doi.org/10.12957/reuerj.2018.33874.

32. Pinto LSS, Oliveira IMP, Pinto ESS, Leite CBC, Melo ADN, Deus MCBR. Women's protection public policies: evaluation of health care for victims of sexual violence. Ciên. Saúde Colet. [Internet]. 2017 [cited 2018 Jul 06]; 22(5):1501-08. DOI: https://doi.org/10.1590/1413-81232017225.33272016. 